\title{
Low-frequency waves in magnetic reconnection
}

\author{
CHAI LiHui ${ }^{1}$, LI Yi $^{1 *}$, WANG Shui ${ }^{1} \&$ SHEN Chao ${ }^{2}$ \\ ${ }^{1}$ School of Earth and Space Sciences, University of Science and Technology of China, Hefei 230026, China; \\ ${ }^{2}$ National Space Science Center, Chinese Academy of Sciences, Beijing 100190, China
}

\begin{abstract}
The characteristics of low-frequency waves in magnetic reconnection are studied using two-dimensional hybrid simulation code. In a coordinate system moving with fluid, the time series of a magnetic field perpendicular to the magnetic reconnection plane, $B_{y}$, is transformed into the power spectrum via fast Fourier transformation, while the wave propagation direction and polarization are determined by minimum variance analysis of the electric field. The results show that low-frequency Alfvén ion-cyclotron waves dominate the reconnection area. These waves have frequencies $0-1 \Omega_{p}$ (where $\Omega_{p}$ is the local proton gyro frequency) and all are left-handed circularly polarized. Among these waves, large-amplitude turbulence, with frequencies of $0-0.6 \Omega_{\mathrm{p}}$ and isotropic propagation, dominates the outflow regions. This can cause the reversal of $B_{y}$ in the quadrupole structure. In the inflow regions, dominant waves, propagating mainly parallel to the ambient magnetic field, have higher frequencies and smaller amplitudes. The frequency of the main peak of wave energy is usually higher than $0.5 \Omega_{\mathrm{p}}$.
\end{abstract}

magnetic reconnection, low-frequency waves, Alfvén-ion cyclotron waves, Hall quadrupole structure

Citation: $\quad$ Chai L H, Li Y, Wang S, et al. Low-frequency waves in magnetic reconnection. Chin Sci Bull, 2012, 57: 1461-1466, doi: 10.1007/s11434-011-4910-4

Magnetic reconnection plays an important role in space plasma physics. It can efficiently transfer and transform the material, momentum, and energy of plasmas. There is much eruptive activity in space plasmas and interaction between the solar wind and Earth's magnetosphere that relate to magnetic reconnection. The classical magnetohydrodynamics (MHD) model with finite resistivity cannot explain such quick energy release because of its low reconnection rate. In recent years, it has been found that the Hall term in the generalized Ohm law has an important effect on collisionless plasmas [1] owing to the contribution of a remarkable increase in the reconnection rate. Research has shown that when the spatial scale of the current sheet $L_{0}$ is larger than the ion inertial length $d_{i}=c / \omega_{p i}$ (where $c$ is the speed of light and $\omega_{p i}$ is the ion plasma frequency), ions and electrons move together following magnetic lines, the MHD model is appropriate and the waves are mainly Alfvén waves; when $L_{0}$ is smaller than $d_{i}$, ions decouple with magnetic lines

*Corresponding author (email: lyi@ustc.edu.cn) while electrons continue to follow them, the separated ions and electrons conduct Hall current, and the out-of-plane component of the magnetic field $B_{y}$ has a Hall quadrupole structure; and when $L_{0}$ is smaller than the electron inertial length $d_{e}$, electrons also decouple from magnetic lines, and the electron dynamics become important $[2,3]$.

Drake [4] firstly studied the dispersive characteristics and plasma waves of collisionless magnetic reconnection, and found that the whistler-wave mode affects magnetic reconnection. Xiao et al. [5] proposed a new electron acceleration scenario of trapped electrons near magnetic null points driven by the whistler-mode waves. Rogers et al. [6] investigated the relation between the reconnection rate and dispersive kinetic Alfvén and whistler waves. Their results show that the wave-and-particle interaction is important at larger scales and that the kinetic Alfvén and whistler waves affect the structure of the dissipation region individually at different parameter scales. Li et al. [7] studied the low-frequency waves in Hall MHD magnetic reconnection with various plasma $\beta$ (where $\beta$ is the ratio of the thermal pres- 
sure to magnetic pressure), and found that waves near the magnetic separatrices behave as whistler waves. Wang et al. [8] investigated the effects of Hall current and the electron pressure gradient and found that (1) when there is no guiding field, the reconnection layer supports obliquely propagating Alfvén-whistler waves, and the reconnection dynamics are controlled by the Hall current waves, and (2) when the guiding field is equal to or larger than the ambient field, the reconnection layer supports kinetic Alfvén waves, and the reconnection dynamics are controlled by the electron pressure gradient. Zhou et al. [9] discussed linear dispersion properties and waves of the plasma current sheet, using 2.5dimensional collisionless and incompressible MHD models with a full electron pressure tensor. Their results show that there are Alfvén waves and ion acoustic waves in the longwavelength region $\left(k d_{i}<1\right)$ and fast magneto sonic-kinetic Alfvén waves and obliquely propagating Alfvén-whistler waves in the short-wavelength region $\left(k d_{i}>1\right)$. Zhang et al. [10] studied the roles of the initial current carrier in the distribution of field-aligned current employing a three-dimensional Hall MHD simulation and found that Alfvén waves propagate anti-parallel to the magnetic field lines.

There have been many theoretical and observational studies on waves with ion cyclotron frequency [11-14]. Hu et al. [15] employed a two-dimensional hybrid code to simulate electromagnetic ion cyclotron (EMIC) waves in a dipole magnetic field, and found that waves along the magnetic equator are generated with dominant left-handed polarization and propagate along the magnetic field toward the ionosphere, and the inhomogeneity of the magnetic field cause the wavefronts to travel oblique to the local magnetic field. When the wave vector turns perpendicular to the magnetic field at high magnetic latitudes, the initially lefthanded waves become linearly polarized. Chaston et al. [16] investigated the characteristics and generation mechanisms of EMIC waves in the near-Earth magnetotail. Bogdanov et al. [17] statistically investigated ion cyclotron waves in the Earth's magnetotail during Cassin's Earth swing-by. They found a maximum in the frequency distribution of the waves at about $0.5 \Omega_{\mathrm{p}}$ (where $\Omega_{\mathrm{p}}$ is the proton cyclotron frequency) and a peak at linear polarization. Nykyri et al. [18] investigated the cluster observations of magnetic field fluctuations in the high-altitude cusp. They found many clear peaks in the wave power around the ion cyclotron frequency, and the regions of enhanced magnetic field fluctuations appear to be associated with plasma flows possibly originating from a lobe reconnection site.

Alfvén ion cyclotron waves in space plasmas have been widely investigated because they can accelerate particles through cyclotron resonance. Broughton et al. [19] studied ultra-low-frequency waves and associated wave vectors observed in the plasma sheet boundary layer by cluster, and discussed the relation between the waves and ion heating. Mecheri et al. [20] studied beam-driven electromagnetic instabilities near the ion-cyclotron frequency in a coronal funnel. Voitenko et al. [21] discussed the kinetic excitation mechanisms for ion-cyclotron kinetic Alfvén waves in the solar corona, solar wind, and Earth's magnetosphere. Chen et al. [22] presented exact solutions to the dispersion equation for MHD waves with short-wavelength modification. Kinetic Alfvén waves have also been investigated extensively because of their non-zero parallel electric field [23-26].

We employed hybrid simulation code developed by Swift and Lin [27] to investigate the characteristics of low-frequency waves in magnetic reconnection. First, we study the space distributions of waves after the reconnection becomes quasi-steady. The characteristics of wave spectra and propagation directions in different regions of magnetic reconnection are studied employing a fast Fourier transform (FFT) and the minimum analysis method (MAM) respectively. Additionally, the effects of large-scale turbulence on the distribution of quadrupole $B_{y}$ are studied.

\section{Simulation model}

Two-dimensional hybrid simulation code considering the Hall current and electron inertial term treats the electrons as massless fluid, and the ions as discrete particles. The magnetic field $\boldsymbol{B}$ is given by Faraday's law:

$$
\frac{\partial \boldsymbol{B}}{\partial t}=-\nabla \times \boldsymbol{E}
$$

The electric field is derived from the electron momentum equation:

$$
\boldsymbol{E}=-\boldsymbol{u}_{e} \times \boldsymbol{B}-v\left(\boldsymbol{u}_{e}-\boldsymbol{u}_{i}\right)-r \frac{\mathrm{d} \boldsymbol{u}_{e}}{\mathrm{~d} t} .
$$

The electron flow velocity is obtained from Ampere's law:

$$
\boldsymbol{u}_{e}=\boldsymbol{u}_{i}-\frac{\nabla \times \boldsymbol{B}}{\alpha n} .
$$

The particle velocity of ions is derived from the ion momentum equation:

$$
\frac{\mathrm{d} \boldsymbol{v}_{i}}{\mathrm{~d} t}=\boldsymbol{E}+\boldsymbol{v}_{i} \times \boldsymbol{B}-v\left(\boldsymbol{u}_{i}-\boldsymbol{u}_{\boldsymbol{e}}\right) .
$$

Here $n$ is the ion number density, $v$ is the collision frequency, $\boldsymbol{v}_{\boldsymbol{i}}$ is the particle velocity of an ion, and $\boldsymbol{u}_{\boldsymbol{e}}$ and $\boldsymbol{u}_{\boldsymbol{i}}$ are the bulk flow velocities of electrons and ions. Additionally, $(\alpha n)^{-1 / 2}=d_{i} / L_{0}$, where $L_{0}$ is the unit length in the simulation, and $d_{i}$ is the ion inertial length. $r$ is the efficiency of the electron inertial effect. Here we take $\alpha=2 / 75, r=1 / 2000$. In the equations, the time, length, velocity, density, temperature, and magnetic field are normalized by $\Omega_{0}^{-1}, d_{i}, v_{A 0}, n_{0}$, $T_{i 0}, B_{0}$, where $\Omega, v_{A}, T_{i}, B$ are the ion gyrofrequency, Alfvén velocity, ion temperature and magnetic field, and the subscript " 0 " indicates initial values.

In this paper, we are only interested in the waves arising from magnetic reconnection instead of the triggering pro- 
cess of reconnection. Therefore, a finite resistivity is imposed at the center of the simulation domain, $(x, z)=(0,0)$, to trigger and control the reconnection. This simulation is for a spontaneous reconnection. The spatial profile of the resistivity corresponds to a collision frequency:

$$
v=v_{0} \mathrm{e}^{-\left(x^{2}+z^{2}\right) / \delta^{2}},
$$

where $v_{0}=2 \Omega_{0}$ and $\delta$ is the half width of the initial current sheet.

In two-dimensional Cartesian coordinates $(x, z)$, the initial profile is given by the Harris static solution. When $t=0$, the magnetic field and density are assumed to be

$$
\begin{gathered}
\boldsymbol{B}_{x}(x, z)=B_{0 x} \tanh [z / \delta], \\
\boldsymbol{B}_{y}(x, z)=B_{y 0}, \\
n(x, z)=n_{0}\left\{1+\frac{1}{\beta_{0}}\left[1-\left(\frac{B_{x 0}^{2}}{B_{0}^{2}}\right) \tanh ^{2}\left(\frac{z}{\delta}\right)-\frac{B_{y 0}^{2}}{B_{0}^{2}}\right]\right\},
\end{gathered}
$$

where $B_{0}^{2}=B_{x 0}^{2}+B_{y 0}^{2}$ is the initial total magnetic field and $\beta_{0}$ is the plasma rate of ion thermal pressure to magnetic pressure. In this paper, the parameters used are $B_{x 0}=2, B_{y 0}=$ $0, n_{0}=150, \beta_{0}=0.04, \delta=0.75$.

At the boundaries $z= \pm L_{z}, B_{z}$ is set to zero. At the boundaries $x= \pm L_{x}$, the magnetic field is extrapolated on the basis of zero curl and divergence of the field. The plasma flow velocity is free.

\section{Simulation results}

\subsection{Formation of the quasi-steady reconnection}

Figure 1 shows the variation in system energy. The total energy $E_{T}=E_{T 0}+E_{\text {in }}=E_{P}+E_{B}+E_{\text {out }}$, where $E_{P}$ is the kinetic energy of particles, $E_{B}$ is the total magnetic energy, $E_{\mathrm{in}}$ is the input energy of the inflows at the boundaries $z= \pm L_{z}$, and $E_{\text {out }}$ is the output energy of the outflows at the boundaries $x= \pm L_{x}$. It is seen that the total energy $E_{T}$ increases linearly because of the input energy at the boundaries. A decrease in magnetic energy $E_{B}$ with an increase in kinetic energy $E_{P}$ indicates that the magnetic energy is converted into the kinetic energy of particles during the reconnection. In the simulation, the magnetic reconnection starts at $t=0$ and begins to accelerate particles. The reconnection then reaches saturation at $t=50 \Omega_{0}^{-1}$ and continues. Massive particles that are accelerated by the magnetic reconnection interact with the background particles and pile up at the top of beams, forming bubble-like piles that move toward the boundaries $x= \pm L_{x}$. The piles move out of the simulation region during $t=180-200 \Omega_{0}^{-1}$. Because of the loss of massive particles during this time, the output energy of the system increases tremendously and both the magnetic energy and kinetic energy decrease. The piles have moved completely out of the region by $t=200 \Omega_{0}^{-1}$ and the magnetic reconnection process

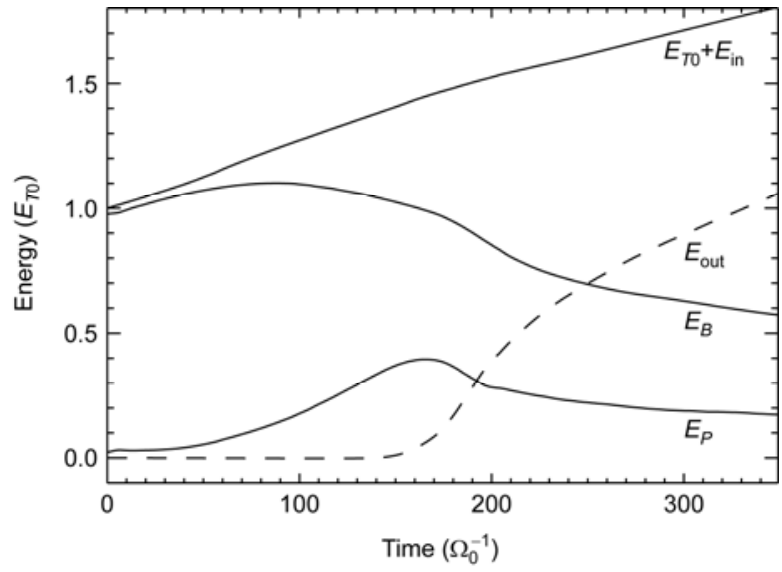

Figure 1 Variation in energy with time.

becomes quasi-steady. In the process of forming a quasisteady state, magnetic reconnection accelerates particles and the accelerated particles interact with background particles and form tremendous instabilities that generate various wave modes. Because of various wave-particle interactions and wave-wave interactions, the whole process presents a chaotic and nonlinear evolution phase. When the reconnection stabilizes, the waves became more ordered. Therefore, the data taken during the quasi-steady process at $t=300$ $350 \Omega_{0}^{-1}$ are chosen to investigate the characteristics of waves.

\subsection{Spatial distribution of waves of quasi-steady reconnection}

Wave energy is defined as the average value of the fluctuation in energy of the magnetic field in an interval of time:

$$
E_{\text {wave }}=\frac{1}{t} \int_{t}(\boldsymbol{B}-\langle\boldsymbol{B}\rangle)^{2} \mathrm{~d} t,
$$

where $\langle\boldsymbol{B}\rangle$, the average value of the magnetic field, represents the background magnetic field. Figure 2 shows the spatial distribution of the wave energy on the right side of the simulation region at $t=300-350 \Omega_{0}^{-1}$; the black lines are the magnetic lines. It is seen that the wave energy is concentrated mainly in the outflow region.

To gain a clearer perspective of the spatial distribution of waves, the contour distribution of $B_{y}$ at $t=300 \Omega_{0}^{-1}$ is shown in Figure 3. It is clear that there is a classical quadrupole structure of $B_{y}$ and there is much large-scale turbulence in the outflow regions and many waves in the inflow region. These distribution characteristics of waves and turbulence confirm the spatial distribution of wave energy in Figure 2.

\subsection{Characteristics of waves in the different regions of magnetic reconnection}

To determine the characteristics of waves in different 


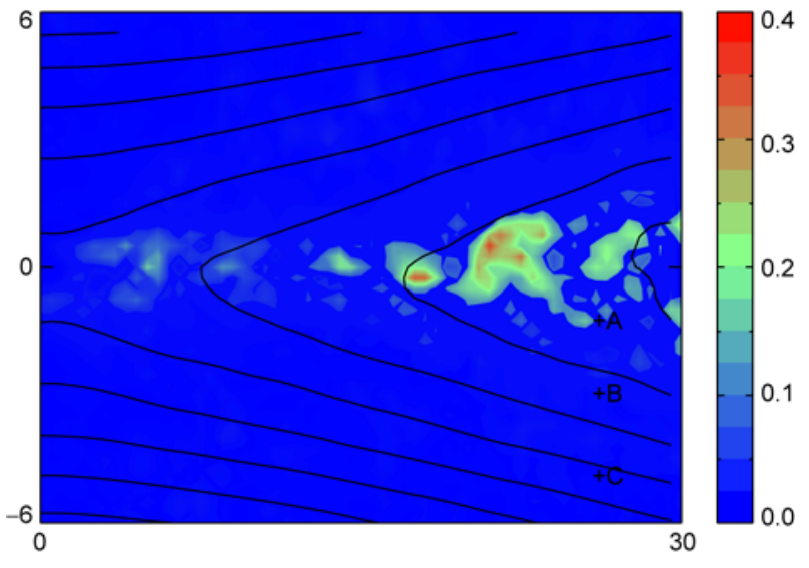

Figure 2 Spatial distribution of the wave energy.

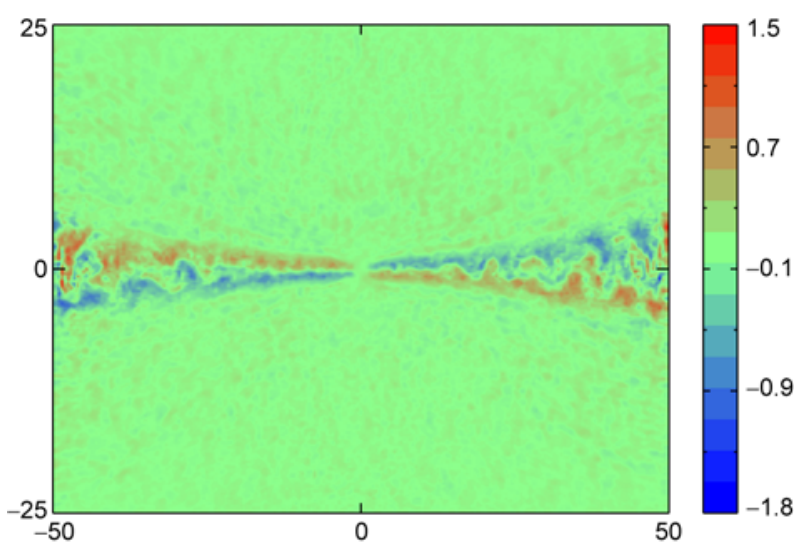

Figure 3 Contours of $B_{y}$ at $t=300 \Omega_{0}^{-1}$.

regions of magnetic reconnection, three points are chosen from the three different regions for study. The locations of the points are marked with " + " in Figure 2. Point $A$ is in the outflow region, point $B$ is on the separatrix, and point $C$ is in the inflow region. The characteristics of waves are studied in three fluid-moving coordinate systems that have origins at the three points.

(i) Wave spectra. The FFT is employed to analyze the 4096 data of the out-of-plane magnetic field $B_{y}$, which are recorded in the moving coordinate system at $t=300-341 \Omega_{0}^{-1}$. Figure 4(a) shows the spectra of $B_{y}$ at points $A, B$ and $C$, where the $x$ axis is the frequency in units of local proton gyrofrequency $\Omega_{\mathrm{p}}$ and the $y$ axis is the energy in units of initial total energy. It is seen that the frequencies of waves are mainly distributed at $0-1 \Omega_{\mathrm{p}}$. The frequency of the wave in the outflow regions is lower $\left(0-0.6 \Omega_{\mathrm{p}}\right)$ and the amplitude is larger. The frequency of the wave increases and the amplitude decreases when the location of waves moves from the outflow region toward the inflow region. In the inflow region, the frequency of the wave energy peak often exceeds $0.5 \Omega_{\mathrm{p}}$. These findings indicate that the low-frequency waves of magnetic reconnection are mainly Alfvén waves and ion cyclotron waves, and that turbulence is abundant in the outflow region.

(ii) Propagation directions of waves. The electric field $\boldsymbol{E}$, from which the average background has been removed, is analyzed employing the MAM to obtain the covariance matrix of the time series of the three electric field components $E_{x}, E_{y}, E_{z}$. The covariance matrix's eigenvector corresponding to its smallest eigenvalue is the shortest principal axis of the variance ellipsoid, which is also the propagation direction $\boldsymbol{k}$ of the wave.

The above method is used to analyze the time series of the electric field $\boldsymbol{E}$ at points A, B and C and thus determine the propagation directions of waves in different regions of magnetic reconnection. Figure 4(b) shows the distribution of the angle between wave propagation direction $\boldsymbol{k}$ and the $x$ axis, here $x$ axis is quasi-parallel with the ambient magnetic field in the inflow regions and quasi-perpendicular with the ambient magnetic field in the outflow regions. It is seen that the propagation directions of waves in the outflow regions are isotropic and those in the inflow regions and near the separatrices are along the ambient magnetic field or in the opposite direction.

(iii) Polarizations of waves. The components of the electric field $E_{i}$ and $E_{j}$ are the projections of the electric field $\boldsymbol{E}$ on the plane perpendicular to the wave propagation direction $\boldsymbol{k}$. Figure 4(c) shows hodographs of $E_{i}$ and $E_{j}$ at the three points $\mathrm{A}, \mathrm{B}$ and $\mathrm{C}$. It is seen that the polarizations of waves throughout the region of magnetic reconnection are left-handed. This indicates that low-frequency waves of magnetic reconnection are composed of waves that are on the left-handed branch of Alfvén waves and ion cyclotron waves.

It needs to be mentioned that the polarizations of waves are mainly left-handed but there are some reverse or nonclassical polarizations, especially in the outflow and in the vicinity of the magnetic separatrices. The reasons for such polarizations are assumed to be the turbulence in the outflow regions and the errors in determining locations of the moving coordinate system due to the large transverse velocity between outflow and inflow regions.

\section{Discussion and conclusions}

\subsection{Alfvén ion cyclotron waves produced by magnetic reconnection}

In this paper, a hybrid simulation code is employed to investigate the low-frequency waves of magnetic reconnection. It is found that in the outflow regions, the frequencies of waves are lower, the amplitudes of waves are larger, and the propagation directions are isotropic, and the polarizations are left-handed except for some reverse or nonclassical polarizations. This indicates that there is massive turbulence accompanying Alfvén ion cyclotron waves in the outflow regions. The speeding-particle beams that are generated by the acceleration of magnetic reconnection interact 
(a)

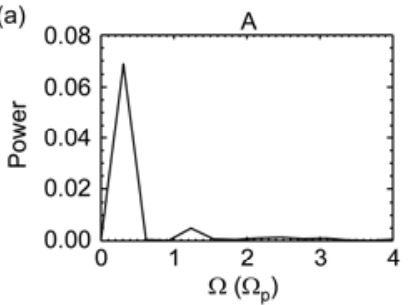

(b)

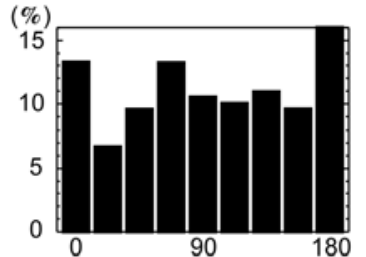

(c)

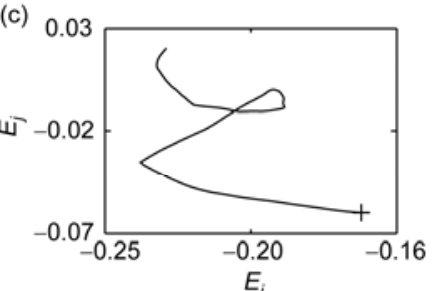

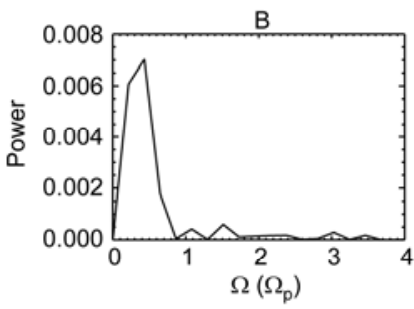
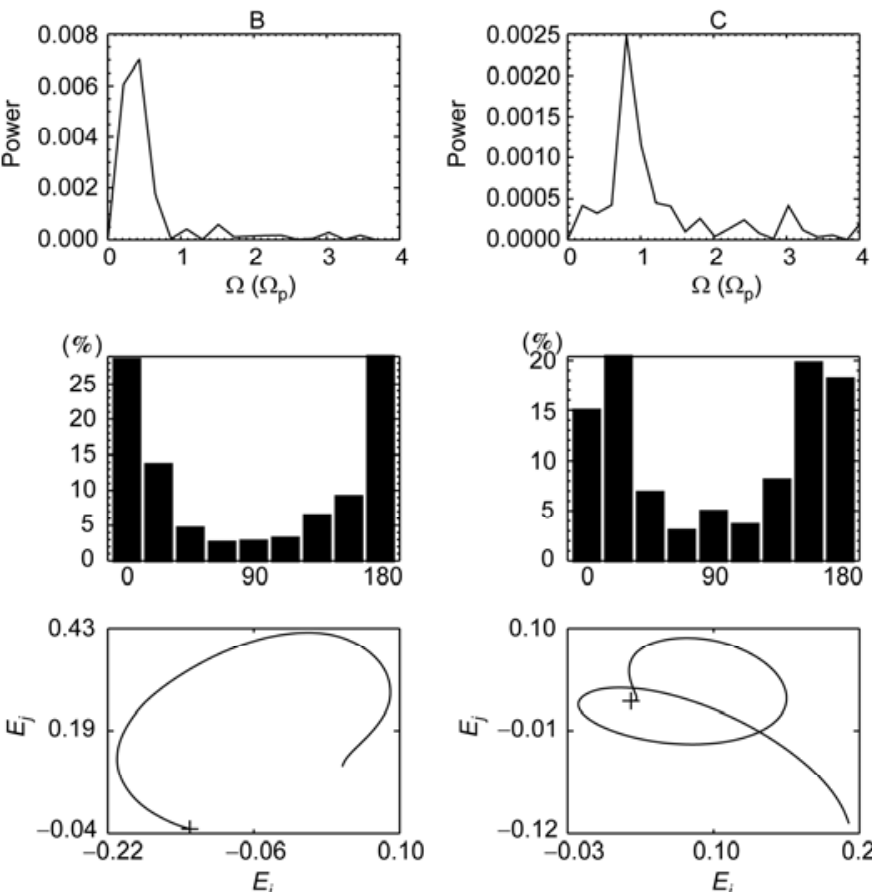
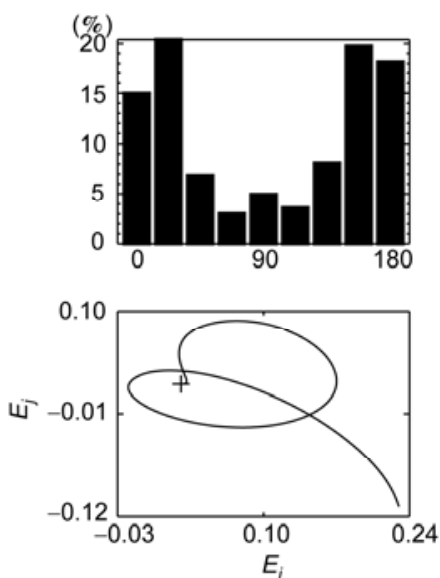

Figure 4 Comparisons among points A, B and C in terms of (a) the power spectrum of $B_{y}$, (b) the angle between the wave vector $\boldsymbol{k}$ and $x$-axis, and (c) the hodograph of the electric field, where + indicates the beginning point.

with background particles along the magnetic field, and induce the EMIC instabilities and various shear instabilities. These instabilities produce massive Alfvén ion cyclotron waves and other wave modes, and thus produce turbulence. The massive waves in the outflow regions imply that there are abundant kinetic waves. In the vicinity of separatrices and inflow regions, the frequencies of waves are higher than those in the outflow regions, waves propagate parallel with magnetic field lines, and the polarization of waves is lefthanded. This indicates that there are mainly left-handed Alfvén and ion cyclotron waves.

Left-handed waves are a majority in our study results. In the inflow regions, waves propagate basically parallel with the direction of the magnetic field, and the left-handed polarization is apparent. In the outflow regions, the directions of wave propagation are generally isotropic, and polarization is left-handed with some complex constructions such as reverse or non-classical polarizations. Most of these results are consistent with the results of Hu et al. [15]. Their investigation of EMIC waves indicated that left-handed EMIC waves transform gradually to linear waves propagating perpendicular to the magnetic field during propagation along the inhomogeneous magnetic field. The results match observations of both left-handed and right-handed waves in the Earth's tail [16] and cusp [18]. Whistler waves are not observed in our simulation probably because the hybrid simulation accentuates ion kinetic effects and is more appropriate to deal with ion inertial scales and low-frequency processes.

\subsection{Reproduction of observations of inverted quadru- pole structure}

The quadrupole distribution of the out-of-plane magnetic field $B_{y}$ is the classical structure of Hall magnetic reconnection. It is also the condition for identification of Hall magnetic reconnection. Past observations have confirmed the existence of the Hall quadrupole structure [28]. However, there remain observed quadrupole structures that are inconsistent with Hall theory. Zhang et al. [10] pointed out that most investigators had ignored these phenomena in past discussions, and they reproduced the inverted quadrupole structure by employing the ion as the carrier of current. They analyzed the observation by cluster SC3 at 2003-9-19 23:1024:00, and found that $37.3 \%$ of the observed data belong to an inverted quadrupole structure. In our investigation, we find that the large-scale turbulence arising from magnetic reconnection also forms an inverted quadrupole structure of $B_{y}$.

As shown in the above analyses of wave characteristics in section 2.3 and by the contours of $B_{y}$ (Figure 3), there is massive turbulence in the outflow regions. Such turbulence affects the distribution of the quadrupole structure of $B_{y}$ to form an inverted quadrupole structure. Figure 5 plots $B_{y}$ along three tracks, which are the neutral line (a), a line parallel to the neutral line (b) and a line perpendicular to the neutral line (c). It is seen that there is an inverted quadrupole structure of $B_{y}$ at many points.

We statistically analyzed the points having an inverted quadrupole structure of $B_{y}$ in the outflow regions. The critical 

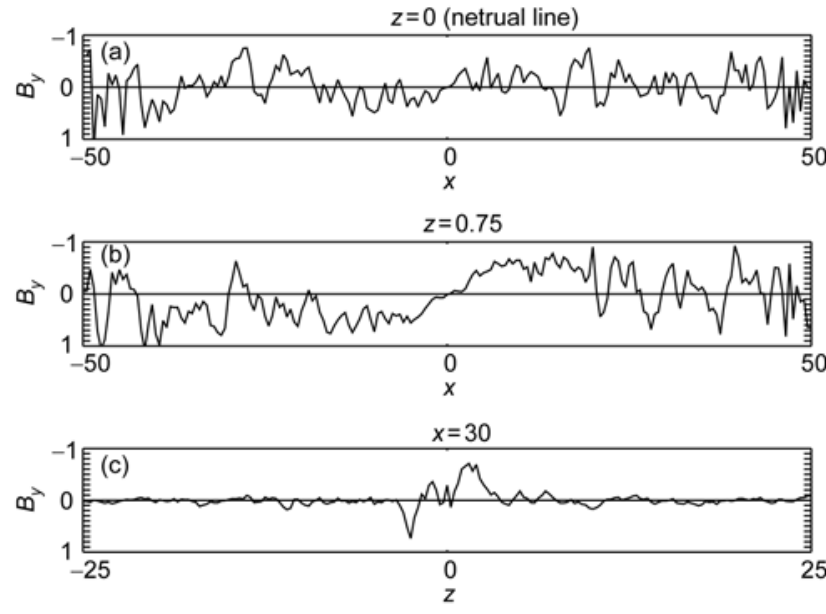

Figure $5 B_{y}$ along the neutral line and lines parallel and perpendicular to the neutral line.

values for judgments of signs/directions of $B_{y}$ are taken as \pm 0.1 , which avoids the effects of small-scale waves. It is found that $16 \%$ of data points of the outflow regions are in regions of inverted quadrupole structure. This result explains the reversal of quadrupole structures often observed in the vicinity of dispersive regions of magnetic reconnection.

Our results are summarized as follows.

(1) In outflow regions, propagation directions of waves are isotropic, polarizations are mainly left-handed, the wave energy is high, and wave frequencies are mainly $0-0.6 \Omega_{\mathrm{p}}$. There is abundant turbulence accompanying Alfvén ion cyclotron waves in the outflow regions.

(2) In the inflow regions and near the magnetic separatrices, the propagation directions of waves are quasi-parallel to the magnetic field, polarization is left-handed, the frequencies of waves are higher than those in outflow regions, and the frequency of a wave's energy peak is often higher than $0.5 \Omega_{\mathrm{p}}$. The waves in the inflow regions are mainly Alfvén ion cyclotron waves.

(3) The large-scale turbulences in the outflow regions can affect the Hall distribution of $B_{y}$ to form the inverted quadrupole structure, which agrees with observations.

This work was supported by the National Natural Science Foundation of China (41074120, 40974101), the Chinese Academy of Sciences (KJCX2-YW-N28), the Fundamental Research Funds of State Key Laboratory of Space Weather and the National Basic Research Program of China (2011CB811404).

1 Shay M A, Drake J F, Rogers B N, et al. Alfvenic collisionless magnetic reconnection and the Hall term. J Geophys Res, 2001, 106: 3759-3772

2 Lu Q M, Wang R S, Xie J L, et al. Electron dynamics in collisionless magnetic reconnection. Chin Sci Bull, 2011, 56: 1174-1181

$3 \mathrm{Lu} \mathrm{S}, \mathrm{Lu} \mathrm{Q} \mathrm{M}$, Cao Y, et al. The effects of the guide field on the structures of electron density depletions in collisionless magnetic reconnection. Chin Sci Bull, 2011, 56: 48-52
4 Drake J F. Magnetic Reconnection: A Kinetic Treatment. Physics of the Magnetopause. Washington: American Geophysical Union, 1995. $155-165$

5 Xiao F L, Zong Q G, Pu Z Y, et al. Electron acceleration by whistler-mode waves around the magnetic null during 3D reconnection. Plasma Phys Control Fusion, 2010, 52: 052001

6 Rogers B N, Denton R E, Drake J F, et al. Role of dispersive waves in collisionless magnetic reconnection. Phys Rev Lett, 2001, 87: 195004

7 Yi L, Jin S P, Yang H G, et al. Numerical study of low frequency wave in Hall MHD reconnection with various plasma $\beta$. Chin J Space Sci, 2007, 27: 96-103

8 Wang X G, Bhattacharjee A, Ma Z W. Collisionless reconnection: Effects of Hall current and electron pressure gradient. J Geophys Res, 2000, 105: 27633-27648

9 Zhou G C, Cao J B, Wang D J, et al. Low-frequency waves in collisionless plasma current sheet. Acta Phys Sin, 2004, 53: 2644-2653

10 Zhang X G, Pu Z Y, Ma Z W, et al. Roles of initial current carrier in the distribution of field-aligned current in 3-D Hall MHD simulations. Sci China Ser E: Tech Sci, 2008, 51: 323-336

11 Tsurutani B T, Dasgupta B, Arballo J K, et al. Magnetic field turbulence, electron heating, magnetic holes, proton cyclotron waves, and the onsets of bipolar pulse (electron hole) events: A possible unifying scenario. Nonlinear Proc Geoph, 2003, 10: 27-35

12 Sundkvist D, Vaivads A, Andre M, et al. Multi-spacecraft determination of wave characteristics near the proton gyrofrequency in highaltitude cusp. Ann Geophys, 2005, 23: 983-995

13 Sundkvist D, Krasnoselskikh V, Shukla P K, et al. In situ multi-satellite detection of coherent vortices as a manifestation of Alfvenic turbulence. Nature, 2005, 436: 825-828

14 Shoji M, Omura Y, Tsurutani B T, et al. Mirror instability and L-mode electromagnetic ion cyclotron instability: Competition in the Earth's magnetosheath. J Geophys Res, 2009, 114: A10203

$15 \mathrm{Hu}$ Y, Denton R E. Two-dimensional hybrid code simulation of electromagnetic ion cyclotron waves in a dipole magnetic field. $\mathrm{J}$ Geophys Res, 2009, 114: A12217

16 Chaston C C, Hu Y D, Fraser B J. Electromagnetic ion cyclotron waves in the near-Earth magnetotail. J Geophys Res, 1999, 104: 6953-6971

17 Bogdanov A T, Glassmeier K H, Musmann G, et al. Ion cyclotron waves in the Earth's magnetotail during CASSINI's Earth swing-by. Ann Geophys, 2003, 21: 2043-2057

18 Nykyri K, Cargill P J, Lucek E, et al. Cluster observations of magnetic field fluctuations in the high-altitude cusp. Ann Geophys, 2004, 22: 2413-2429

19 Broughton M C, Engebretson M J, Glassmeier K H, et al. Ultralow-frequency waves and associated wave vectors observed in the plasma sheet boundary layer by Cluster. J Geophys Res, 2008, 113: A12217

20 Mecheri R, Marsch E. Coronal ion-cyclotron beam instabilities within the multi-fluid description. Astron Astrophys, 2007, 474: 609-615

21 Voitenko Y, Goossens M. Kinetic excitation mechanisms for ion- cyclotron kinetic Alfven waves in Sun-Earth connection. Space Sci Rev, 2003, 107: 387-401

22 Chen L, Wu D J. Exact solutions of dispersion equation for MHD waves with short-wavelength modification. Chin Sci Bull, 2011, 56: 955-961

23 Wu D J, Huang G L, Wang D Y, et al. Solitary kinetic Alfven waves in the two-fluid model. Phys Plasmas, 1996, 3: 2879-2884

24 Wu D J, Fang C. Sunspot chromospheric heating by kinetic Alfven waves. Astrophys J, 2007, 659: L181-L184

25 Wu D J. Dissipative solitary kinetic alfven waves and electron acceleration in the solar corona. Space Sci Rev, 2005, 121: 333-342

26 Chai L H, Yi L. Solitary kinetic Alfven waves in adiabatic process. Phys Plasmas, 2009, 16: 122309

27 Lin Y, Swift D W. A two-dimensional hybrid simulation of the magnetotail reconnection layer. J Geophys Res, 1996, 101: 19859-19870

28 Borg A L, Oieroset M, Phan T D, et al. Cluster encounter of a magnetic reconnection diffusion region in the near-Earth magnetotail on September 19, 2003. Geophys Res Lett, 2005, 32: L19105

Open Access This article is distributed under the terms of the Creative Commons Attribution License which permits any use, distribution, and reproduction in any medium, provided the original author(s) and source are credited. 